\title{
Maximization of a Second-Degree Polynomial on the Unit Sphere
}

\section{By James W. Burrows*}

I. Introduction. Let $A$ be a hermitian matrix of order $n$, and $a$ be a known vector in $C^{n}$. The problem is to determine which vectors make $\Phi(x)=x^{*} A x-2 \operatorname{Re}\left\{x^{*} a\right\}$ ( ${ }^{*}$ denotes conjugate transpose) a maximum or minimum on the unit sphere $S=\left\{x: x^{*} x=1\right\}$.

[1] considers finding the similarly constrained maximum or minimum of $(x-b)^{*} A(x-b)$ where $b$ is a known vector. We have

$$
\begin{aligned}
(x-b)^{*} A(x-b) & =x^{*} A x-b^{*} A x-x^{*} A b+b^{*} A b \\
& =x^{*} A x-2 \operatorname{Re}\left\{x^{*} A b\right\}+b^{*} A b
\end{aligned}
$$

so with $a=A b$, the problems are seen to be equivalent unless $A$ is singular, in which case our formulation is more general. This formulation also seems to lead to simpler proofs.

II. Computation of Extremal Vectors. Let $U$ be the unitary transformation which diagonalizes $A$, i.e., if $x=U y$, then

$$
x^{*} A x-2 \operatorname{Re}\left\{x^{*} a\right\}=y^{*} U^{*} A U y-2 \operatorname{Re}\left\{y^{*} U^{*} a\right\}=y^{*} \Lambda y-2 \operatorname{Re}\left\{y^{*} c\right\},
$$

where $c=U^{*} a$ and $\Lambda=\operatorname{diag}\left\{\lambda_{1}, \cdots, \lambda_{n}\right\}$, with real $\lambda_{i}$. It is thus equivalent to find the maximum or minimum of

$$
\psi(y)=\sum_{i=1}^{n} \lambda_{i}\left|y_{i}\right|^{2}-2 \operatorname{Re}\left\{\sum_{i=1}^{n} c_{i} \bar{y}_{i}\right\}
$$

with the constraint

$$
\sum_{i=1}^{n}\left|y_{i}\right|^{2}=1
$$

Construct

$$
\chi(y)=\sum_{i=1}^{n} \lambda_{i}\left|y_{i}\right|^{2}-2 \operatorname{Re}\left\{\sum_{i=1}^{n} c_{i} \bar{y}_{i}\right\}-\lambda \sum_{i=1}^{n}\left|y_{i}\right|^{2}
$$

where stationarity with respect to complex $y$ requires that the Lagrange multiplier $\lambda$ be real (cf. [1], p. 30). An extremal vector then satisfies the equation

$$
0=\frac{1}{2} \operatorname{grad} \chi(y)=\Lambda y-c-\lambda y=0
$$

or

$$
\left(\lambda_{i}-\lambda\right) y_{i}=c_{i}, \quad i=1, \cdots, n .
$$

If we solve this formally for $y_{i}$ and substitute into (2.3) we are led to consider the

Received November 12, 1965.

* The author is with the Mathematical Analysis Staff, The Boeing Company, Seattle, Washington. 
real roots of the equation

$$
g(\lambda)=1
$$

with

$$
g(\lambda)=\sum_{i=1}^{n} \frac{\left|c_{i}\right|^{2}}{\left(\lambda-\lambda_{i}\right)^{2}}
$$

A primed summation sign means terms with $c_{i}=0$ are dropped, whatever the value of $\lambda-\lambda_{i}$. Two cases can occur:

Case I. $\lambda$ is a real root of (2.6) and $\lambda \neq \lambda_{i}$ for all $i$. Then (2.5) gives the components of an extremal vector $y_{\lambda}$ associated with $\lambda$.

Case II. For some $k, g\left(\lambda_{k}\right) \leqq 1$. This requires $c_{i}=0$ for all $i$ such that $\lambda_{i}=\lambda_{k}$. To obtain the components of an extremal vector $y_{\lambda_{k}}$ associated with $\lambda_{k}$, solve (2.5) for $y_{i}$ if $\lambda_{i} \neq \lambda_{k}$, then select any $y_{i}$ for $i$ such that $\lambda_{i}=\lambda_{k}$ so that

$$
\sum_{i: \lambda_{i}=\lambda_{k}}\left|y_{i}\right|=1-g\left(\lambda_{k}\right)
$$

Then both (2.5) and the constraint (2.3) are satisfied.

Theorem. Let $\lambda_{j}$ be the largest eigenvalue of $A$ for which $g\left(\lambda_{j}\right) \leqq 1$. Let $\underline{\lambda}$ be the largest root of (2.6) with $\underline{\lambda} \neq \lambda_{i}, i=1, \cdots, n$. The quadratic polynomial $\psi(y)$ is maximized by a vector associated with the larger of $\underline{\lambda}$ and $\lambda_{j}$.

Proof. For real $\lambda \neq \lambda_{i}, i=1, \cdots, n$, let the components of $y_{\lambda}$ be given by (2.5), then

$$
\begin{aligned}
\psi\left(y_{\lambda}\right) & =\sum_{i=1}^{n} \lambda_{i} \frac{\left|c_{i}\right|^{2}}{\left(\lambda_{i}-\lambda\right)^{2}}-2 \operatorname{Re}\left\{\sum_{i=1}^{n} \frac{\left|c_{i}\right|^{2}}{\lambda_{i}-\lambda}\right\} \\
& =\sum_{i=1}^{n}\left|c_{i}\right|^{2}\left[\frac{\lambda_{i}}{\left(\lambda_{i}-\lambda\right)^{2}}-\frac{2}{\lambda_{i}-\lambda}\right] \\
& =\lambda \sum_{i=1}^{n} \frac{\left|c_{i}\right|^{2}}{\left(\lambda-\lambda_{i}\right)^{2}}+\sum_{i=1}^{n} \frac{\left|c_{i}\right|^{2}}{\lambda-\lambda_{i}} \\
& =\lambda g(\lambda)+\sum_{i=1}^{n} \frac{\left|c_{i}\right|^{2}}{\lambda-\lambda_{i}} .
\end{aligned}
$$

If $\lambda$ is a root of $(2.6)$, then

$$
\psi\left(y_{\lambda}\right)=\lambda+\sum_{i=1}^{n} \frac{\left|c_{i}\right|^{2}}{\lambda-\lambda_{i}}
$$

If $\lambda=\lambda_{k}$ and the other conditions of Case II are fulfilled, then the value of $\psi\left(y_{\lambda}\right)$ for $\lambda=\lambda_{k}$ is calculated by priming the summation sign in (2.9) and adding

$$
\lambda_{k} \sum_{i: \lambda_{i}=\lambda_{k}}\left|y_{i}\right|^{2}
$$

We then have

$$
\psi\left(y_{\lambda}\right)=\lambda g(\lambda)+\sum_{i=1}^{n} \lambda \frac{\left|c_{i}\right|^{2}}{\lambda-\lambda_{i}}+\lambda_{k} \sum_{i: \lambda_{i}=\lambda_{k}}\left|y_{i}\right|^{2}=\lambda+\sum_{i=1}^{n} \lambda-\left.c_{i}\right|^{2} .
$$

When $\lambda \neq \lambda_{i}$ for all $i,(2.11)$ is the same as (2.10). Therefore, (2.11) is true for all 
extremal vectors. To complete the proof, let $\mu, \nu$ be two values of $\lambda$ which satisfy the conditions of either Case I or Case II, and suppose $\mu>\nu$. Then

$$
\begin{aligned}
\psi\left(y_{\mu}\right)-\psi\left(y_{\nu}\right) & =\mu+\sum_{i=1}^{n} \frac{\left|c_{i}\right|^{2}}{\mu-\lambda_{i}}-\nu-\sum_{i=1}^{n} \frac{\left|c_{i}\right|^{2}}{\nu-\lambda_{i}} \\
& =\mu-\nu+\sum_{i=1}^{n}\left|c_{i}\right|^{2}\left(\frac{1}{\mu-\lambda_{i}}-\frac{1}{\nu-\lambda_{i}}\right) \\
& =(\mu-\nu)\left[1-\sum_{i=1}^{\prime} \frac{\left|c_{i}\right|^{2}}{\left(\mu-\lambda_{i}\right)\left(\nu-\lambda_{i}\right)}\right] \\
& \geqq(\mu-\nu)\left[\frac{1}{2} g(\mu)+\frac{1}{2} g(\nu)-\sum_{i=1}^{\prime} \frac{\left|c_{i}\right|^{2}}{\left(\mu-\lambda_{i}\right)\left(\nu-\lambda_{i}\right)}\right] \\
& \geqq \frac{1}{2}(\mu-\nu) \sum_{i=1}^{n}\left|c_{i}\right|^{2}\left[\left(\mu-\lambda_{i}\right)^{2}+\frac{2}{\left(\nu-\lambda_{i}\right)^{2}}-\frac{2}{\left(\mu-\lambda_{i}\right)\left(\nu-\lambda_{i}\right)}\right] \\
& \geqq 0 .
\end{aligned}
$$

Therefore, $\psi\left(y_{\lambda}\right)$ increases for increasing $\lambda$ which satisfy either Case I or Case II. This proves the theorem; a similar statement about the minimum of the polynomial is easily proven.

III. An Application. Let $(x, y, z)$ be the position vector of a target in a coordinate system attached to a rolling ship and $(\dot{x}, \dot{y}, \dot{z})$ the target's inertial velocity vector in the same coordinates. Consider the angular accelerations of a gun tracking this target. The gun has the usual two degrees of freedom: a train axis perpendicular to the deck and an elevation axis perpendicular to the train axis. Let $\theta$ be the train angle. The parts of the train angular acceleration $\ddot{\theta}$ which contain the target velocity are

$$
\begin{aligned}
\ddot{\theta}(\dot{x}, \dot{y}, \dot{z})=2\left(x^{2}+y^{2}\right)^{-2}\{ & x y\left(\dot{x}^{2}-\dot{y}^{2}\right)-\dot{x} \dot{y}\left(x^{2}-y^{2}\right) \\
& \left.+\dot{R}\left[z\left(y^{2}-x^{2}\right) \dot{x}-2 x y z \dot{y}\right]\right\}+2 \dot{R} x\left(x^{2}+y^{2}\right)^{-1} \dot{z},
\end{aligned}
$$

where $\dot{R}$ is the roll rate (assumed to be about the $x$-axis). The last term can be recognized as a component of the Coriolis acceleration. The remaining terms can be computed by considering the relative motion in a nonrotating system (i.e., take two derivatives of $y=x \tan \theta$ ). The problem of maximizing the entire expression as a function of $\dot{x}, \dot{y}, \dot{z}$ with $\dot{x}^{2}+\dot{y}^{2}+\dot{z}^{2}=1$ and fixed $x, y, z, \dot{R}$ is of the type considered, with $A$ singular. In fact,

$$
A=2\left(x^{2}+y^{2}\right)^{-2}\left(\begin{array}{ccc}
x y & -\frac{1}{2}\left(x^{2}-y^{2}\right) & 0 \\
-\frac{1}{2}\left(x^{2}+y^{2}\right) & -x y & 0 \\
0 & 0 & 0
\end{array}\right)
$$

and

$$
a^{*}=-\left(x^{2}+y^{2}\right)^{-2} \dot{R}\left(z\left(y^{2}-x^{2}\right),-2 x y z, x\left(x^{2}+y^{2}\right)\right) .
$$

Further computation yields

$$
\lambda_{1}=-\left(x^{2}+y^{2}\right)^{-1}, \quad \lambda_{2}=\left(x^{2}+y^{2}\right)^{-1}, \quad \lambda_{3}=0
$$




$$
\begin{aligned}
& U==\left[2\left(x^{2}+y^{2}\right)\right]^{-1 / 2}\left(\begin{array}{ccc}
x-y & x+y & 0 \\
x+y & y-x & 0 \\
0 & 0 & {\left[2\left(x^{2}+y^{2}\right)\right]^{1 / 2}}
\end{array}\right) \\
& c^{*}=a^{*} U=-(2)^{-1 / 2}\left(x^{2}-y^{2}\right)^{-3 / 2} \dot{R}(-z(x+y), z(y-x), \\
&\left.x\left[2\left(x^{2}+y^{2}\right)\right]^{1 / 2}\right) .
\end{aligned}
$$

Therefore,

$$
\begin{aligned}
&\left(x^{2}+y^{2}\right) \psi=y_{2}{ }^{2}-y_{1}{ }^{2}+ \dot{R}(2)^{1 / 2}\left(x^{2}+y^{2}\right)^{-1 / 2} \\
& \cdot\left(-z(x+y) y_{1}+z(y-x) y_{2}+x\left[2\left(x^{2}+y^{2}\right)\right]^{1 / 2} y_{3}\right) .
\end{aligned}
$$

After neglecting the fixed factor $x^{2}+y^{2}$,

$$
\frac{2 g(\lambda)}{\dot{R}^{2}}=\frac{z^{2}(x+y)^{2}}{\left(x^{2}+y^{2}\right)(\lambda+1)^{2}}+\frac{z^{2}(y-x)^{2}}{\left(x^{2}+y^{2}\right)(\lambda-1)^{2}}+\frac{2 x^{2}}{\lambda^{2}} .
$$

In the general case, when none of the numerators are zero, the problem is solved by finding the largest real root of $(3.8)$ with $g(\lambda)=1$. Classical root calculation procedures, such as Newton's method, should encounter no difficulty. If one or more of the numerators are zero, the computation is simpler. For example, if $z=0$, $g(\lambda)=\dot{R}^{2} x^{2} / \lambda^{2}$ and Case I applies if $\lambda=|\dot{R} x| \geqq 1$. Then $y_{1}=y_{2}=0, y_{3}= \pm 1$; if $|\dot{R} x|<1$, then Case II applies and $y_{1}=0, y_{2}=\left(1-\dot{R}^{2} x^{2}\right)^{1 / 2}, y_{3}=\dot{R} x$. The geometric interpretation of this is that the Coriolis term predominates for large $x$ values.

1. George E. Forsythe \& Gene H. Golub, Maximizing a second-degree polynomial on the unit sphere, Tech. Rep. CS16, Stanford University Computer Science Department, Stanford, Calif., 1965.

\title{
Questions Concerning Khintchine's Constant and the Efficient Computation of Regular Continued Fractions
}

\author{
By John W. Wrench, Jr. and Daniel Shanks
}

Let $x$ be a real number whose regular continued fraction is given by

$$
x=a_{0}+\frac{1}{a_{1}}+\frac{1}{a_{2}}+\frac{1}{a_{3}}+\ldots,
$$

with $a_{0}$ an integer, and $a_{1}, a_{2}, a_{3}, \cdots$ positive integers. Let

$$
G_{n}(x)=\left(a_{1} \cdot a_{2} \cdot a_{3} \cdot \cdots \cdot a_{n}\right)^{1 / n} .
$$

Then Khintchine's famous theorem states that, for almost all $x$,

$$
\operatorname{Lim}_{n \rightarrow \infty} G_{n}(x)=K,
$$

Received January 24, 1966. 